\title{
Serial Electron Crystallography: New Developments for Data Collection and Analysis
}

Robert Bücker ${ }^{1}$, Pascal Hogan-Lamarre ${ }^{2}$, Pedram Mehrabi ${ }^{3}$, Eike Schulz ${ }^{3}$, Günther Kassier ${ }^{4}$ and R. J. Dwayne Miller ${ }^{5}$

${ }^{1}$ Centre for Structural System Biology, University of Hamburg, Hamburg, Germany, United States, ${ }^{2}$ Department of Physics, University of Toronto, Toronto, ON, Canada, United States, ${ }^{3}$ Hamburg Centre for Ultrafast Imaging, HARBOR, University of Hamburg, Hamburg, Germany, United States, ${ }^{4}$ Max Planck Institute for the Structure and Dynamics of Matter, Hamburg, Germany, United States, ${ }^{5}$ Departments of Physics and Chemistry, University of Toronto, Toronto, ON, Canada, United States

Serial electron diffraction (SerialED) (Smeets, Zou and Wan, 2018; Bücker et al., 2020) is an emerging threedimensional electron diffraction (3D-ED) method (Gemmi et al., 2019; Nannenga and Gonen, 2019), where data from a large ensemble of macromolecular or small-molecule nano-crystals is merged into a high-resolution structure solution. Like in serial X-ray crystallography at synchrotrons and X-ray free-electron lasers (Mehrabi et al., 2020), SerialED is typically performed in the limit of rapidly acquired single or dose-fractionated diffraction shots per diffracting volume, minimizing deleterious effects of radiation damage. However, the flexibility of a scanning TEM (S/TEM) enables a much broader range of data collection strategies, blurring the lines between serial crystallography (SX), scanning electron nano-diffraction (SEND), and massively automated rotation electron diffraction (RED). Furthermore, the flexibility of SerialED along with the large volume and unique nature of the obtained diffraction data, that is, purely two-dimensional reciprocal-space slices of unknown orientation, call for new approaches for data processing (Jiang et al., 2009; Smeets and Wan, 2017; Bücker, Hogan-Lamarre and Miller, 2020; Gevorkov et al., 2020).

We present results from a range of biological and organic samples such as in-situ grown proteins, covalent and metal-organic frameworks (COFs/MOFs), highlighting the excellent performance of SerialED in obtaining structures of highly radiation sensitive crystals, as well as robustness against contaminated and inhomogeneous specimens. Also, considerations regarding the optimal data collection and analysis strategies for a given sample, as well as various methods of determining unknown unit cells from two-dimensional snapshot data will be discussed. The recently developed software package diffractem (Bücker, Hogan-Lamarre and Miller, 2020), is introduced, which allows for easy pre-processing and data reduction of many-terabytes SerialED data sets, and wraps CrystFEL (White et al., 2012) for the tasks of crystal orientation determination and merging of partially excited reflections. Finally, an outlook on ongoing projects towards time-resolved SerialED, and serial/scanning ED on filamentous samples, is given. 

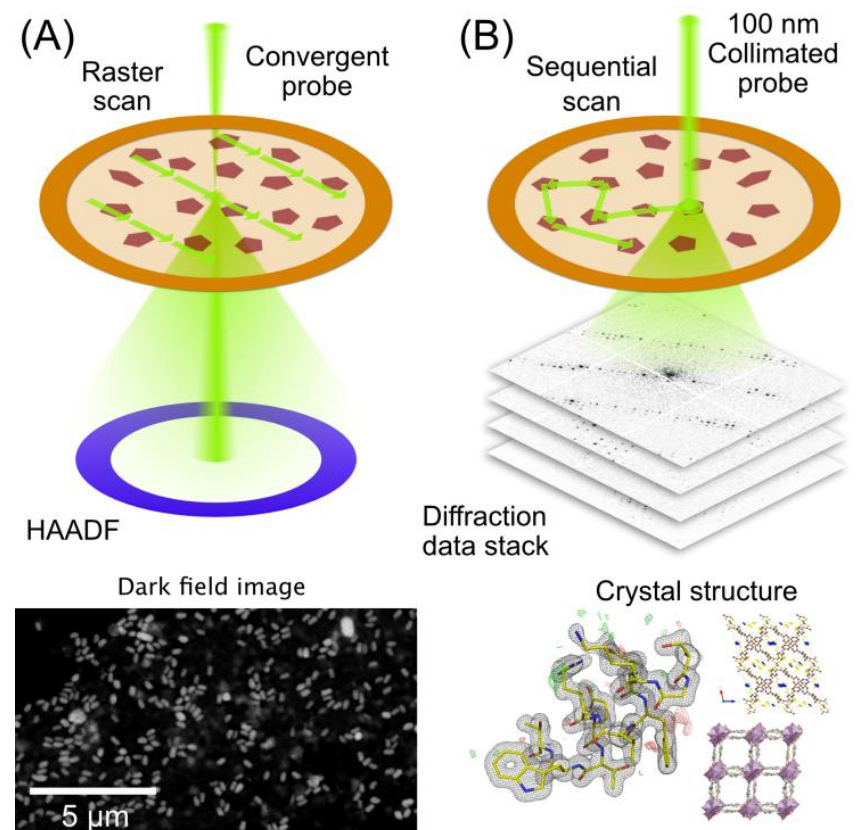

$\rightarrow 5$ im

Dark field image

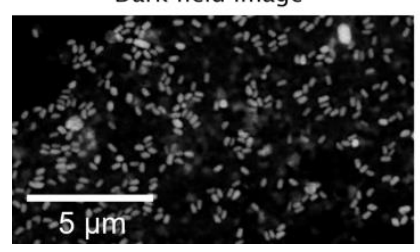

Figure 1. Scheme of a SerialED experiment. (A) A sample region containing nano-crystals is screened in STEM low-mag real-space imaging. (B) Diffraction patterns from each crystal are acquired in rapid succession by steering the beam to each crystal. From the resulting patterns, the crystal structure is reconstructed. 

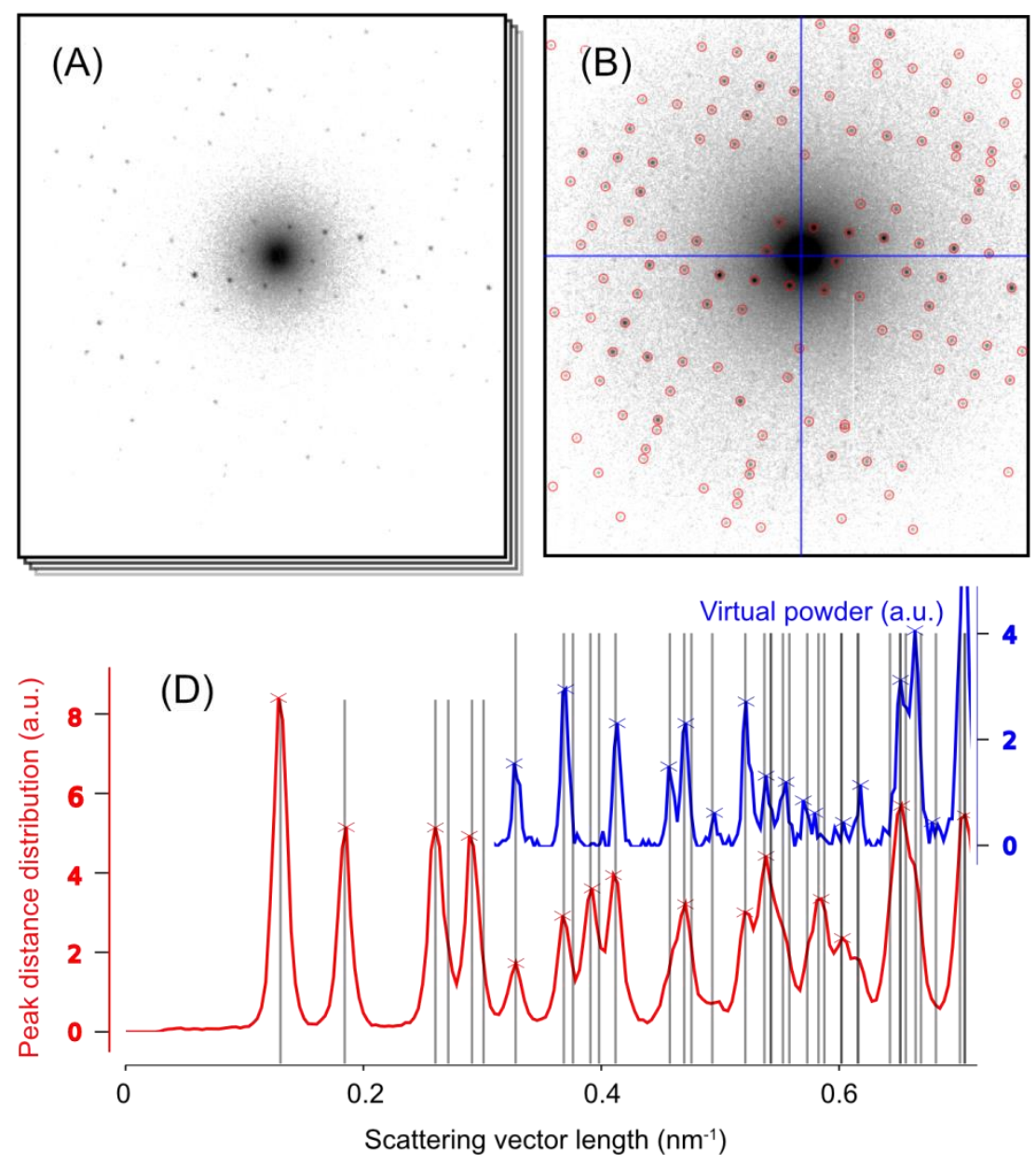
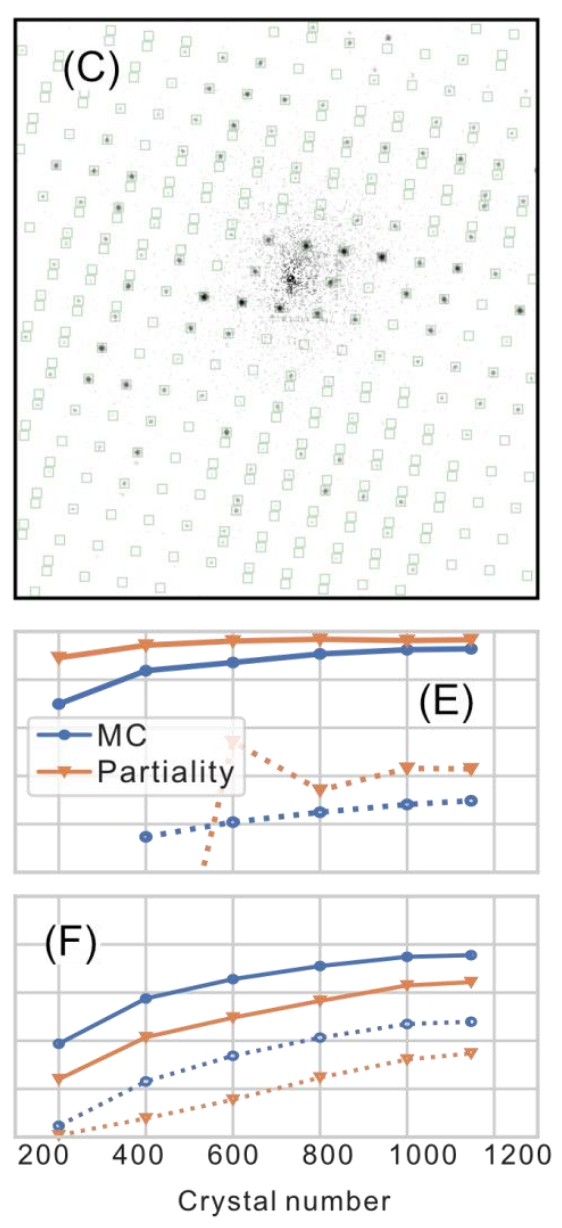

Figure 2. Steps of SerialED analysis. (A) Schematic stack of dose-fractionated frames comprising a single diffraction pattern snapshot. (B) Aggregated pattern, i.e., partial sum over frames in (A), with found peaks and pattern center annotated. (C) Background-corrected pattern after indexing, with Bragg reflection positions predicted from the found crystal unit cell and pose annotated. (A-C) are shown on the same logarithmic scale. (D) Histogram of radial peak positions (blue) and pair-wise peak distances (red) aggregated over many tetragonal lysozyme nano-crystals. The data allows to refine the unit cell parameters by fitting predicted d-spacings (grey lines). (E) Validation metrics $\mathrm{CC} 1 / 2$ and completeness for a proteincrystal dataset increase as a function of merged crystal numbers. A clear difference is found between merging strategies with (orange) or without (blue) taking into account partiality.

References

Bücker, R. et al. (2020) 'Serial protein crystallography in an electron microscope', Nature Communications, 11, p. 996. doi: 10.1038/s41467-020-14793-0.

Bücker, R., Hogan-Lamarre, P. and Miller, R. J. D. (2020) 'Serial Electron Diffraction Data Processing with diffractem and CrystFEL', arXiv:2011.02977

Gemmi, M. et al. (2019) '3D Electron Diffraction: The Nanocrystallography Revolution', ACS Central Science, 5(8), pp. 1315-1329. doi: 10.1021/acscentsci.9b00394. 
Gevorkov, Y. et al. (2020) 'pinkIndexer - a universal indexer for pink-beam X-ray and electron diffraction snapshots', Acta Crystallographica Section A Foundations and Advances, 76(2). doi: $10.1107 / \mathrm{S} 2053273319015559$.

Jiang, L. et al. (2009) 'Unit-cell determination from randomly oriented electron-diffraction patterns', Acta Crystallographica Section D: Biological Crystallography, 65(7), pp. 625-632. doi: 10.1107/S0907444909003163.

Mehrabi, P. et al. (2020) 'Serial femtosecond and serial synchrotron crystallography yield data of equivalent quality: a systematic comparison', bioRxiv, p. 2020.08.21.257170. doi: 10.1101/2020.08.21.257170.

Nannenga, B. L. and Gonen, T. (2019) 'The cryo-EM method microcrystal electron diffraction (MicroED)', Nature Methods, 16(5), pp. 369-379. doi: 10.1038/s41592-019-0395-x.

Smeets, S. and Wan, W. (2017) 'Serial electron crystallography: Merging diffraction data through rank aggregation', Journal of Applied Crystallography, 50(3), pp. 885-892. doi: 10.1107/S1600576717005854. Smeets, S., Zou, X. and Wan, W. (2018) 'Serial electron crystallography for structure determination and phase analysis of nanocrystalline materials', Journal of Applied Crystallography, 51(5), pp. 1-12. doi: $10.1107 /$ S1600576718009500.

White, T. A. et al. (2012) 'CrystFEL : a software suite for snapshot serial crystallography', Journal of Applied Crystallography, 45(2), pp. 335-341. doi: 10.1107/S0021889812002312. 